\title{
Diffuse large B-cell lymphoma with pulmonary and cerebral involvement: A case report
}

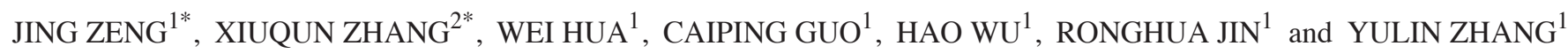 \\ ${ }^{1}$ Department of Infectious Diseases, Capital Medical University Affiliated Beijing You An Hospital, Beijing 100069; \\ ${ }^{2}$ Department of Hematology, Nanjing Medical University Affiliated Nanjing \\ First Hospital, Nanjing, Jiangsu 210006, P.R. China
}

Received January 18, 2018; Accepted July 12, 2018

DOI: $10.3892 / \mathrm{mco} .2018 .1673$

\begin{abstract}
Primary diffuse large B-cell lymphoma (DLBCL) is the most common type of non-Hodgkin lymphoma; however, the involvement of the lung and central nervous system (CNS) in patients with DLBCL is rare. Furthermore, patients with DLBCL rarely exhibit specific clinical symptoms, which may delay definitive diagnosis. The present study reports the case of a 42-year-old man suffering from primary DLBCL with concurrent pulmonary and cerebral involvement. The patient suffered from human immunodeficiency virus infection and presented with symptoms including dry cough, thoracalgia, intermittent mild fever and mild headache. Thoracic computed tomography scans revealed multiple pulmonary masses, and brain magnetic resonance imaging scans revealed nodules in the left frontal cortex and bilateral basal ganglia. A percutaneous lung needle biopsy test confirmed the diagnosis of DLBCL. In addition, positron emission tomography revealed the involvement of other parts of the body in DLBCL. The aim of the present study was to present the clinical, radiological and histological characteristics of the patient, which may aid physicians in diagnosing pulmonary and CNS involvement in DLBCL.
\end{abstract}

\section{Introduction}

High-grade non-Hodgkin lymphoma (NHL) is considered to represent one of three acquired immune deficiency syndrome (AIDS)-defining malignancies (1), among which diffuse large B-cell lymphoma (DLBCL) is the most common, accounting for $31 \%$ of all NHL cases $(2,3)$. However, primary non-Hodgkin

Correspondence to: Dr Yulin Zhang or Dr Ronghua Jin, Department of Infectious Diseases, Capital Medical University Affiliated Beijing You An Hospital, 8 Xitoutiao Road, Beijing 100069, P.R. China

E-mail: zhangyulin1968@126.com

E-mail:jin_eagle@sina.com

${ }^{*}$ Contributed equally

Key words: diffuse large B-cell lymphoma, pulmonary, cerebral, tuberculosis lymphoma of the lung is uncommon and accounts for only $0.4 \%$ of all malignant lymphomas (4). The majority of primary pulmonary lymphomas (PPL) are of low grade, of the mucosa-associated lymphoid tissue type, and primary pulmonary DLBCL is extremely rare (5). Primary central nervous system lymphoma (PCNSL) is a rare tumor of the brain that accounts for $4 \%$ of newly diagnosed patients with CNS malignancies (6,7); however, the majority of patients with PCNSL have the highly aggressive diffuse large B-cell subtype. In the present study, the case of a patient suffering from DLBCL with concurrent pulmonary and cerebral involvement is reported. This is rare in clinical practice and, considering that its clinical symptoms are non-specific, prompt diagnosis often proves difficult (8).

\section{Case report}

A 42-year-old man presented with a 2-week history of dry cough and thoracalgia accompanied by intermittent mild fever and headaches; however, no night sweats or weight loss were reported. The patient had a history of sexual contact with men. A total of 18 months prior to the presentation, the patient was diagnosed with pulmonary tuberculosis (TB) in a specialized hospital in China following the development of fever and cough. The results of acid-fast staining on sputum smear analysis and a chest X-ray showing infiltrates in both lungs were consistent with typical TB manifestations (Fig. 1A). Simultaneously, the patient was diagnosed with human immunodeficiency virus (HIV) infection. According to the guideline for adult HIV/AIDS patients with opportunistic infections recommended by the Center for Disease Control and Prevention, Infectious Diseases Society of America and National Institutes of Health (http:// aidsinfo.nih.gov/guidelines), the specialist consultant at the TB specialized hospital empirically prescribed first-line anti-TB treatment for this patient with 2 months of isoniazid, rifampicin, ethambutol and pyrazinamide, followed by 4 months of isoniazid and rifampicin. As a result, the fever and cough were eliminated, and the pulmonary infiltrate was also absorbed (Fig. 1B and C). Thus, according to the guideline, further testing for anti-TB drug sensitivity was not deemed necessary. One month after the aforementioned treatments, lamivudine, tenofovir and efavirenz were administered to the patient as an additional anti-HIV treatment strategy; however, the medication was not 

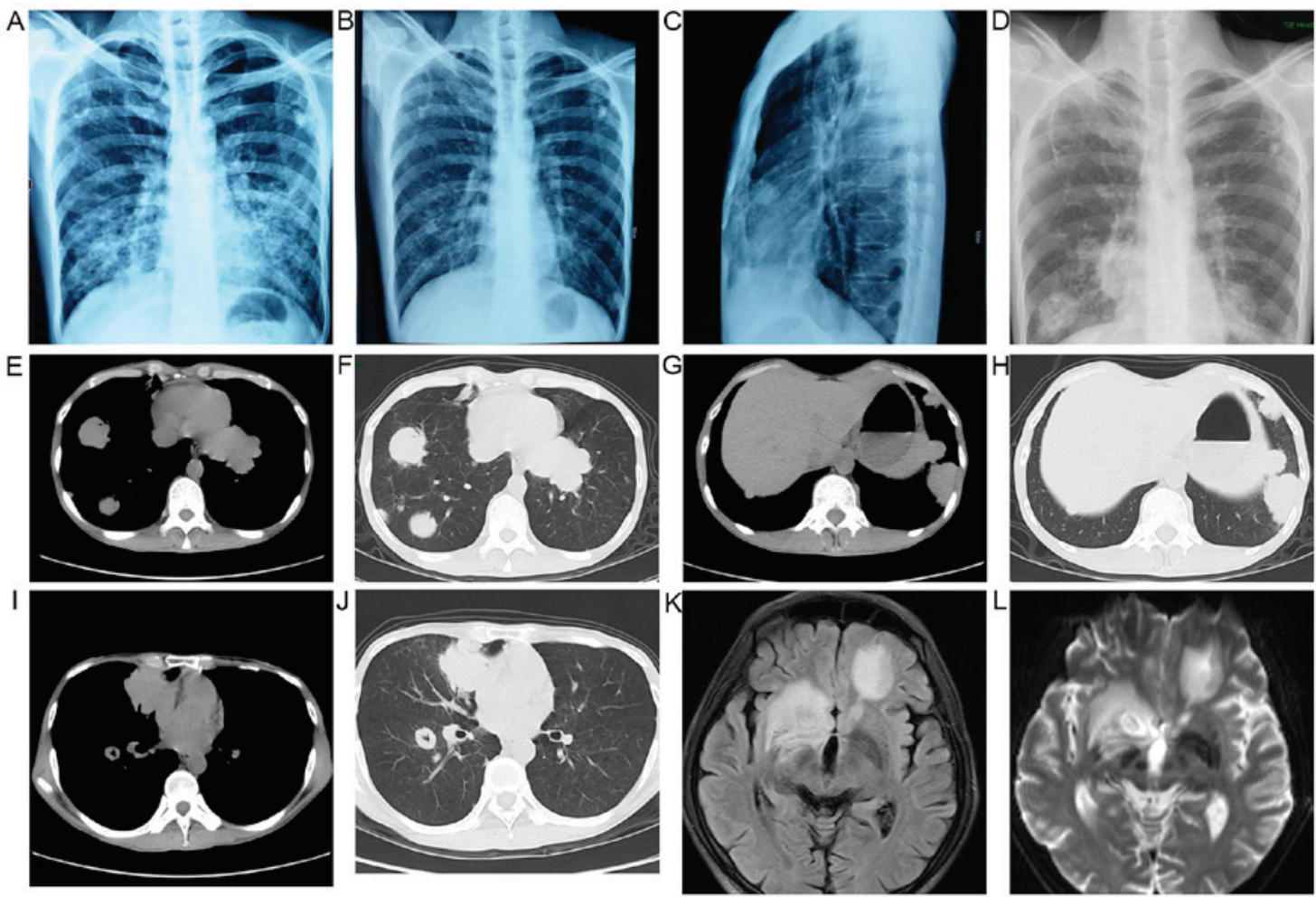

Figure 1. Imaging examinations revealed masses in both lungs and the brain. (A) A chest X-ray revealed infiltrates and calcifications in both lungs (18 months prior to the presentation). (B and C) A chest X-ray performed after 6 months of standard first-line anti-tuberculosis chemotherapy revealed that the pulmonary infiltrate was absorbed. (D) A chest X-ray revealed multiple masses in both lungs. (E-J) Thoracic computed tomography scans revealed multiple nodules and masses in both lungs. (K and L) Brain magnetic resonance imaging revealed nodules in the left frontal cortex and bilateral basal ganglia.

taken regularly and follow-up appointments were also irregular. A physical examination was performed following admittance to the hospital, which revealed bilateral oral leukoplakia and tenderness in the left lower chest. Sputum acid-fast staining analysis did not reveal any signs of TB infection, and no anaerobic bacteria, aerobic bacteria, fungi or Mycobacterium tuberculosis were present in blood culture. The results of the laboratory tests performed on admission are presented in Table I. Lumbar puncture was performed due to the patient suffering from headaches; however, no pathogens were found in the cerebrospinal fluid (CSF). The results of CSF examinations are presented in Table I. A chest X-ray revealed multiple masses in both lungs (Fig. 1D) and a subsequent thoracic computerized tomography (CT) scan confirmed this result (Fig. 1E-J). Brain magnetic resonance imaging (MRI) revealed nodules in the left frontal cortex and the bilateral basal ganglia. (Fig. $1 \mathrm{~K}$ and L). The patient was initially suspected to be suffering from a pulmonary and CNS infection; however, the X-ray and laboratory findings did not support this suspected diagnosis. To determine the composition of the lung and brain masses, a percutaneous lung needle biopsy was performed, revealing large numbers of lymphocytes ranging in size from medium to large, with oval or round nuclei containing fine chromatin and scanty cytoplasm (Fig. 2A-C). Furthermore, immunohistochemistry staining analysis revealed that these results were consistent with a diagnosis of germinal center B-cell-like (GCB) DLBCL (Fig. 2D-G). ${ }^{18} \mathrm{~F}$-labelled fluorodeoxyglucose (FDG) positron emission tomography (PET) revealed that FDG uptake was high in the brain, mediastinum, lungs, right adrenal gland, thoracic vertebrae and ribs (Fig. 3). Based on these results, experienced radiologists suggested a diagnosis of systemic lymphoma with CNS involvement. Then, bone marrow aspiration was performed, and cytological examination revealed normal bone marrow hyperplasia, without the presence of lymphosarcoma cells. Therefore, the patient was diagnosed with stage IV NHL according to the Ann Arbor staging system for lymphoma (9), B group as he displayed one of the systemic B symptoms, including fevers $\left(>38.5^{\circ} \mathrm{C}\right)$, drenching night sweats and/or weight loss ( $>10 \%$ of body weight over 6 months prior to diagnosis). The patient had a poor prognosis due to his high-intermediate risk (score 3) according to international prognostic index: Stage IV, high serum lactate dehydrogenase level, $>1$ extranodal sites (10) and CD4 cell count $<100$ cells $/ \mu 1$ (11). Unfortunately, the patient refused chemotherapy or radiotherapy for the treatment of lymphoma due to his poor economic status and poor prognosis, and discharged himself from the hospital.

\section{Discussion}

The majority of risk factors for the development of lymphoma are associated with altered immune function (12), including infection with HIV, the administration of immunosuppressants for the prevention of organ transplant rejection and severe autoimmune conditions (13). DLBCL is the most common type of NHL in HIV-infected as well as non-HIV infected patients (3). The majority of patients with PPL suffer from marginal zone lymphomas of the mucosa-associated lymphoid tissue type $(14,15)$. DLBCL directly arising from lung tissue is extremely rare, and accounts for $0.4 \%$ of all lymphomas (16). Primary pulmonary DLBCL is frequently misdiagnosed as 
Table I. Laboratory test results on admission.

\begin{tabular}{|c|c|c|}
\hline Test item & Test value & Normal range \\
\hline White blood cell count $\left(10^{9} / 1\right)$ & 2.94 & $3.5-9.5$ \\
\hline Neutrophils (\%) & 73.5 & $40-75$ \\
\hline Lymphocytes (\%) & 15.0 & $20-50$ \\
\hline Hemoglobin (g/l) & 95.0 & $130-175$ \\
\hline Platelets $\left(10^{9} / 1\right)$ & 202 & $125-350$ \\
\hline Blood urea nitrogen (mmol/l) & 4.19 & $2.29-7.0$ \\
\hline Creatinine $(\mu \mathrm{mol} / \mathrm{l})$ & 47.6 & $53-106$ \\
\hline Alanine transaminase (U/l) & 34.9 & $9-50$ \\
\hline Glutamic-oxaloacetic transaminase (U/l) & 39.8 & $15-40$ \\
\hline Total bilirubin $(\mu \mathrm{mol} / \mathrm{l})$ & 4.0 & $5-20$ \\
\hline Direct bilirubin $(\mu \mathrm{mol} / \mathrm{l})$ & 2.6 & $1.7-10$ \\
\hline Albumin $(\mathrm{g} / \mathrm{l})$ & 28.6 & $40-55$ \\
\hline$\beta 2$-microglobulin (mg/l) & 6.08 & $1.09-2.53$ \\
\hline Lactate dehydrogenase (U/1) & 526 & $135-225$ \\
\hline CD4 cell count (cells $/ \mu 1)$ & 42 & $600-800$ \\
\hline Erythrocyte sedimentation rate $(\mathrm{mm} / \mathrm{h})$ & 50 & $<15$ \\
\hline High-sensitivity C-reactive protein (mg/l) & 35 & $0-3$ \\
\hline Procalcitonin (ng/ml) & 1.76 & $<1.0$ \\
\hline Plasma $(1,3) \beta$-D-glucan (pg/ml) & 10 & $<60$ \\
\hline Serum galactomannan antigen & Negative & Negative \\
\hline Cryptococcal antigen & Negative & Negative \\
\hline Anti-EBV-EA IgM antibody & Negative & Negative \\
\hline Anti-EBV-VCA IgM antibody & Negative & Negative \\
\hline Anti-CMV IgM antibody & Negative & Negative \\
\hline Anti-mycoplasma IgM antibody & Negative & Negative \\
\hline Anti-chlamydia $\operatorname{IgM}$ antibody & Negative & Negative \\
\hline EBV DNA (copies/ml) & $<500$ & $<500$ \\
\hline CMV DNA (copies/ml) & $<500$ & $<500$ \\
\hline HIV RNA loads (copies/ml) & $10 \times 10^{6}$ & $<500$ \\
\hline CSF pressure $\left(\mathrm{mmH}_{2} \mathrm{O}\right)$ & 85 & $80-180$ \\
\hline CSF total cell count $\left(10^{6} / 1\right)$ & 0 & $0-8$ \\
\hline CSF protein (g/l) & 0.22 & $0.15-0.45$ \\
\hline CSF glucose (mmol/l) & 2.59 & $2.8-4.5$ \\
\hline CSF chloride (mmol/l) & 120.9 & $120-132$ \\
\hline CSF TOX-IgM & Negative & Negative \\
\hline CSF TOX-IgG & Negative & Negative \\
\hline
\end{tabular}

EBV, Epstein-Barr virus; CMV, cytomegalovirus; EA, early antigen; VCA, viral capsid antigen; HIV, human immunodeficiency virus; CSF, cerebrospinal fluid; TOX, toxoplasma.

pneumonia, TB or lung cancer due to its non-specific clinical symptoms, and definitive diagnosis most often requires invasive lung biopsy $(5,17)$. There has been a report of a patient suffering from pulmonary lymphomatoid granulomatosis who was misdiagnosed as suffering from pulmonary aspergillosis and TB (18).

PCNSL is a rare type of extranodal NHL (19), and congenital or acquired immunodeficiency is the only risk factor for this tumor that has been established to date. Patients with PCNSL have lymphosarcoma cells present in the CNS, which may induce lymphoma relapse (20). Unlike peripheral tumors, cerebral lymphoma is difficult to diagnose by histopathology.
Therefore, MRI and PET-CT examinations can assist in the diagnosis of most CNS lymphomas. The typical findings of PCNSL on MRI are considered to be single lesions (60-70\% of the cases) or multiple lesions (30-40\% of the cases) without necrosis and with a relatively small oedema, which are usually localized in the periventricular space (21). FDG-PET has been shown to provide high accuracy in the differentiation between cerebral lymphomas and infectious lesions in patients with AIDS. Malignant processes tend to exhibit higher uptake compared with infections (22). In the present case, the brain MRI revealed nodules in the left frontal cortex and bilateral basal ganglia, and the FDG-PET revealed high signal in the brain, 

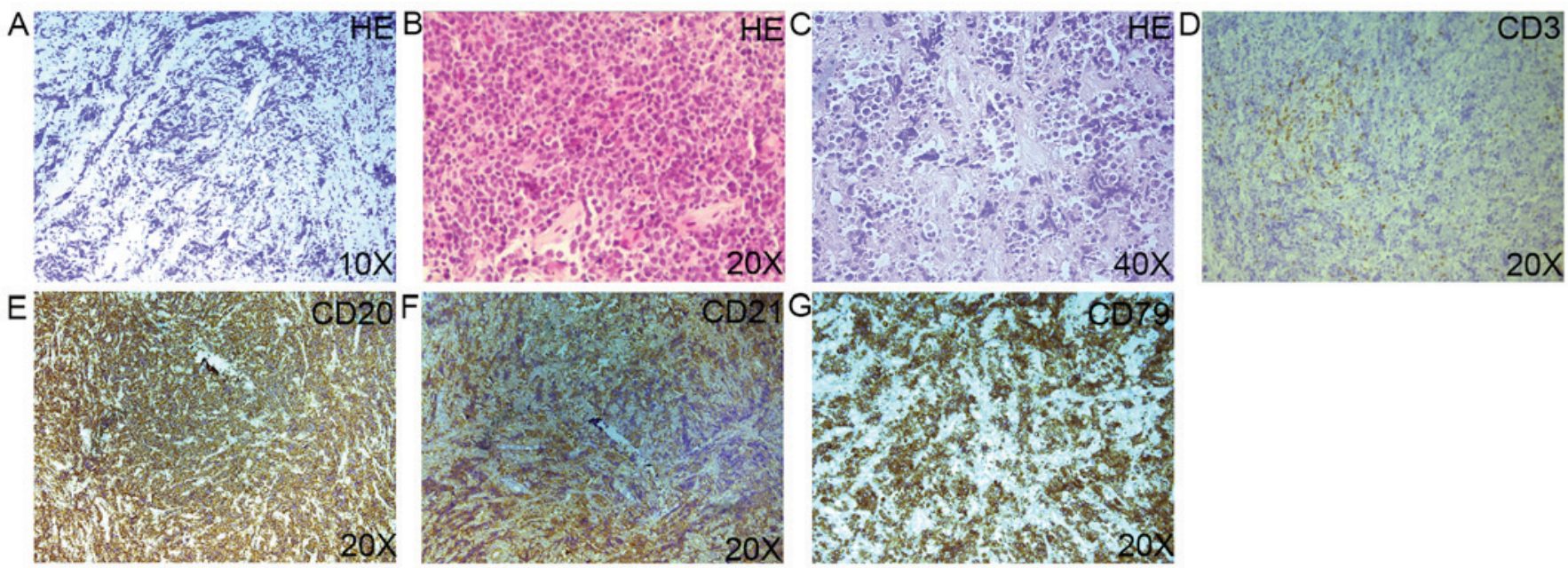

Figure 2. (A-C) Histopathology of the pulmonary biopsy specimen revealed large numbers of lymphocytes ranging in size from medium to large, with oval or round nuclei containing fine chromatin and scanty cytoplasm (hematoxylin and eosin staining). (D-G) Immunohistochemistry of the pulmonary biopsy specimen revealed positive CD3, CD20, CD21, CD79 expression on the surface of the lymphocytes.
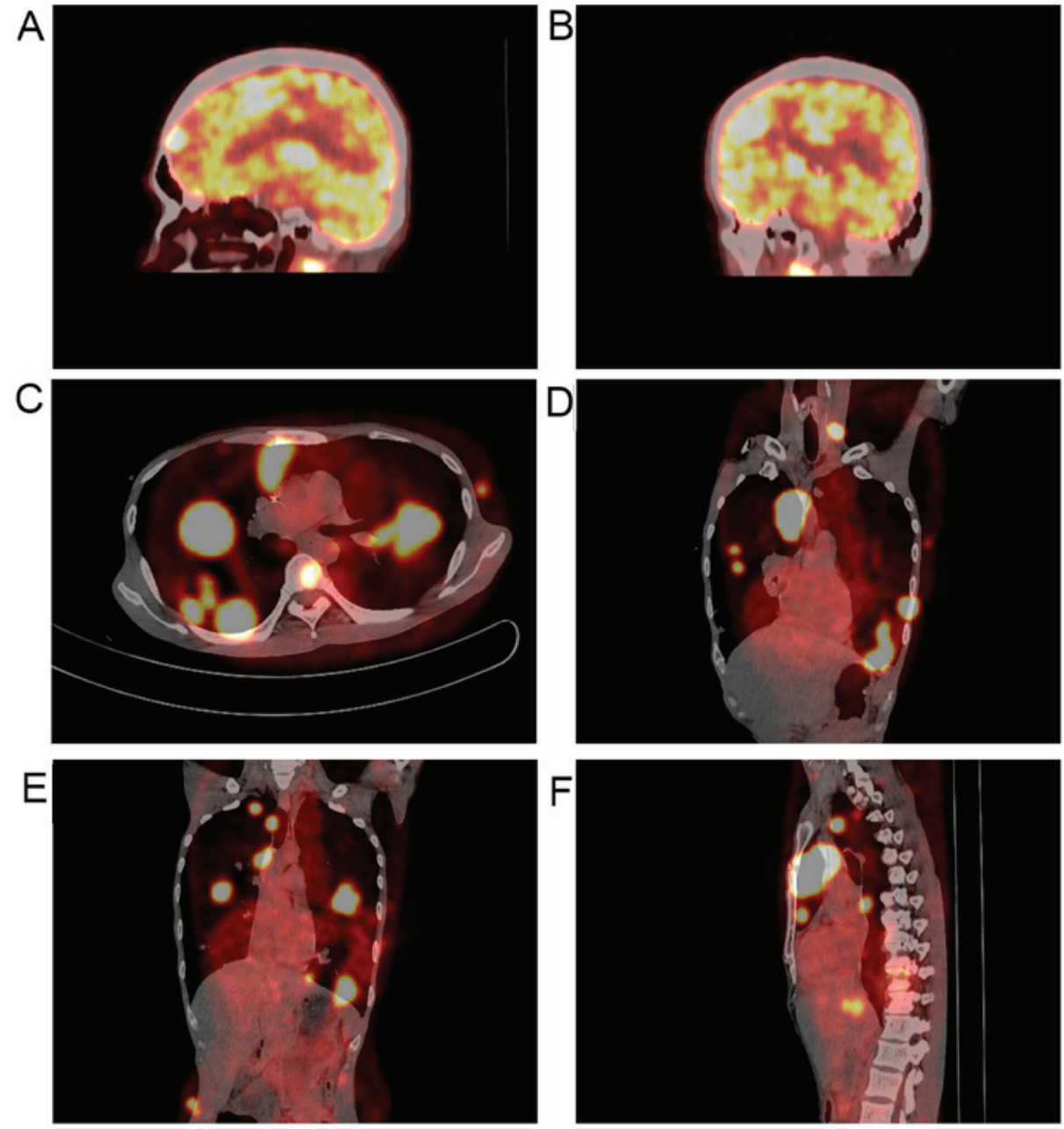

Figure 3. FDG-PET scans revealed numerous high signal tumors. (A-F) PET scan images revealed that FDG uptake was high in the brain, mediastinum, lungs, right adrenal gland, thoracic vertebrae and ribs. FDG, fluorodeoxyglucose; PET, positron emission tomography.

which were considered to be consistent with the MRI and PET characteristics of CNS lymphoma by experienced radiologists based on the NCCN Clinical Practice Guideline in Oncology (www. NCCN.org). Furthermore, other CNS malignancies were excluded, as the simultaneous presence of a systemic lymphoma and another carcinoma in the same patient is extremely rare. The most common pathogenic infections in the brain parenchyma of HIV/AIDS patients are toxoplasma, TB and cryptococcosis. 
The clinical presentation and laboratory tests for the presence of pathogenic microorganisms in the blood and CSF did not confirm the diagnosis of cerebral parenchymal pathogen infection in the present case. Furthermore, the radiographic appearance of the CNS lesions did not support this diagnosis. Therefore, infectious lesions of the brain were not considered in this case.

In conclusion, patients suffering from both pulmonary and nervous system lymphoma are extremely rare. This case study was reported with the aim to increase awareness among physicians regarding the presence of DLBCL with concurrent pulmonary and CNS involvement.

\section{Acknowledgements}

Not applicable.

\section{Funding}

The present study was supported by the National Natural Science Foundation of China (grant no. 81571178).

\section{Availability of data and materials}

Not applicable.

\section{Authors' contributions}

JZ and XZ selected clinical information; WH, CG and HW, provided clinical diagnostic support; RJ and $\mathrm{YZ}$ prepared the manuscript. All authors read and approved the final version of the manuscript.

\section{Ethics approval and consent to participate}

This case report was approved by the Human Subjects Protection Committees of Beijing You An Hospital, Capital Medical University.

\section{Patient consent for publication}

Written informed consent was obtained from the patient regarding the publication of the case details.

\section{Competing interests}

The authors declare that they have no competing interests to disclose.

\section{References}

1. Bower M, Palfreeman A, Alfa-Wali M, Bunker C, Burns F, Churchill D, Collins S, Cwynarski K, Edwards S, Fields P, et al: British HIV association guidelines for HIV-associated malignancies 2014. HIV Med 15 (Suppl 2): S1-S92, 2014.

2. Martelli M,Ferreri A, Agostinelli C, Di Rocco A, Pfreundschuh M and Pileri S: Diffuse large B-cell lymphoma. Crit Rev Oncol Hematol 87: 146-171, 2013.

3. Birendra KC, Afzal MZ, Wentland KA, Hashmi H, Singh S, Ivan $\mathrm{E}$ and Lakhani N: Spontaneous regression of refractory diffuse large B-cell lymphoma with improvement in immune status with ART in a patient with HIV: A case report and literature review. Am J Case Rep 16: 347-352, 2015.
4. Majid N, Kamal el B, Oncology B, Rachid A and Hassan IH: Primary pulmonary lymphoma: About five cases and literature review. Lung India 31: 53-55, 2014.

5. Jiang AG, Gao XY and Lu HY: Diagnosis and management of a patient with primary pulmonary diffuse large B-cell lymphoma: A case report and review of the literature. Exp Ther Med 8: 797-800, 2014.

6. Montesinos-Rongen M, Purschke FG, Brunn A, May C, Nordhoff E, Marcus K and Deckert M: Primary central nervous system (CNS) lymphoma B cell receptors recognize CNS proteins. J Immunol 195: 1312-1319, 2015.

7. Villano JL, Koshy M, Shaikh H, Dolecek TA and McCarthy BJ: Age, gender, and racial differences in incidence and survival in primary CNS lymphoma. Br J Cancer 105: 1414-1418, 2011.

8. Ferraro P, Trastek VF, Adlakha H, Deschamps C, Allen MS and Pairolero PC: Primary non-Hodgkin's lymphoma of the lung. Ann Thorac Surg 69: 993-997, 2000.

9. Lister TA, Crowther D, Sutcliffe SB, Glatstein E, Canellos GP, Young RC, Rosenberg SA, Coltman CA and Tubiana M: Report of a committee convened to discuss the evaluation and staging of patients with Hodgkin's disease: Cotswolds meeting. J Clin Oncol 7: 1630-1636, 1989.

10. Ribera JM, Oriol A, Morgades M, González-Barca E, Miralles P, López-Guillermo A, Gardella S, López A, Abella E and García M; PETHEMA, GELTAMO, GELCAB and GESIDA Groups: Safety and efficacy of cyclophosphamide, adriamycin, vincristine, prednisone and rituximab in patients with human immunodeficiency virus-associated diffuse large B-cell lymphoma: results of a phase II trial. Br J Haematol 140: 411-419, 2008.

11. Straus DJ, Huang J, Testa MA, Levine AM and Kaplan LD: Prognostic factors in the treatment of human immunodeficiency virus-associated non-Hodgkin's lymphoma: Analysis of AIDS clinical trials group protocol 142-low-dose versus standard-dose m-BACOD plus granulocyte-macrophage colony-stimulating factor. National institute of allergy and infectious diseases. J Clin Oncol 16: 3601-3606, 1998.

12. Lin Y, Gustafson MP, Bulur PA, Gastineau DA, Witzig TE and Dietz AB: Immunosuppressive CD14+HLA-DR(low)/monocytes in B-cell non-Hodgkin lymphoma. Blood 117 : 872-881, 2011.

13. Torre LA, Bray F, Siegel RL, Ferlay J, Lortet-Tieulent J and Jemal A: Global cancer statistics, 2012. CA Cancer J Clin 65: 87-108, 2015.

14. Saitoh Y, Ohnishi-Amemiya A, Asano M, Tanaka Y, Yoshizawa S, Fujimoto $\mathrm{H}$, Itoh $\mathrm{Y}$, Nakamura $\mathrm{N}$ and Ohyashiki K: Unique radiological features of two cases of primary pulmonary diffuse large B-cell lymphoma. Thorax 72: 859-860, 2017.

15. Zucca E, Gregorini A and Cavalli F: Management of non-Hodgkin lymphomas arising at extranodal sites. Ther Umsch 67: 517-525, 2010.

16. Xu H, Xu K, Wang R and Liu X: Primary pulmonary diffuse large B-cell lymphoma on FDG PET/CT-MRI and DWI. Medicine (Baltimore) 94: e1210, 2015.

17. Cardenas-Garcia J, Talwar A, Shah R and Fein A: Update in primary pulmonary lymphomas. Curr Opin Pulm Med 21: 333-337, 2015.

18. Xu B, Liu H, Wang B, Zhang H, Wu H, Jin R and Zhang Y: Fever, dry cough and exertional dyspnea: Pulmonary lymphomatoid granulomatosis masquerading as pneumonia, granulomatosis with polyangiitis and infectious mononucleosis. Intern Med 54: 3045-3049, 2015.

19. Batchelor T and Loeffler JS: Primary CNS lymphoma. J Clin Oncol 24: 1281-1288, 2006.

20. Uni M, Kagoya Y, Nannya Y, Nakamura F and Kurokawa M: Central nervous system relapse in patients with diffuse large B-cell lymphoma: Analysis of incidence and prognostic factors. Leuk Lymphoma 56: 1869-1871, 2015.

21. Korfel A and Schlegel U: Diagnosis and treatment of primary CNS lymphoma. Nat Rev Neurol 9: 317-327, 2013.

22. Maza S, Buchert R, Brenner W, Munz DL, Thiel E, Korfel A and Kiewe P: Brain and whole-body FDG-PET in diagnosis, treatment monitoring and long-term follow-up of primary CNS lymphoma. Radiol Oncol 47: 103-110, 2013.

This work is licensed under a Creative Commons Attribution-NonCommercial-NoDerivatives 4.0 International (CC BY-NC-ND 4.0) License. 